\title{
Antisense therapeutics in oncology: current status
}

This article was published in the following Dove Press journal:

OncoTargets and Therapy

3 November 2014

Number of times this article has been viewed

\author{
Ammad Ahmad Farooqi' \\ Zia ur Rehman ${ }^{2}$ \\ Jordi Muntane 3,4 \\ 'Laboratory for Translational \\ Oncology and Personalized Medicine, \\ Rashid Latif Medical College, \\ Lahore, Pakistan; ${ }^{2}$ Department \\ of Biotechnology and Genetic \\ Engineering, Kohat University of \\ Science and Technology (KUST), \\ Kohat, Pakistan; ${ }^{3}$ Department \\ of General Surgery, Institute of \\ Biomedicine of Seville (IBiS), Hospital \\ Universitary "Virgen del Rocío"/CSIC/ \\ University of Seville, Sevilla, Spain; \\ ${ }^{4}$ Centro de Investigación Biomédica \\ en Red de Enfermedades Hepáticas y \\ Digestivas (CIBEREHD o Ciberehd), \\ Instituto de Salud Carlos III, Spain
}

Correspondence: Ammad Ahmad Farooqi

Laboratory for Translational Oncology and Personalized Medicine, RLMC, Lahore, Pakistan

Email ammadahmad638@yahoo.com

\begin{abstract}
There is increasing progress in translational oncology and tremendous breakthroughs have been made as evidenced by preclinical and clinical trials. Data obtained from high-throughput technologies are deepening our understanding about the molecular and gene network in cancer cells and rapidly emerging in vitro and in vivo evidence is highlighting the role of antisense agents as specific inhibitors of the expression of target genes, thus modulating the response of cancer cells to different therapeutic strategies. Much information is continuously being added into various facets of molecular oncology and it is now understood that overexpression of antiapoptotic proteins, oncogenes, oncogenic microRNAs (miRNA), and fusion proteins make cancer cells difficult to target. Delivery of antisense oligonucleotides has remained a challenge and technological developments have helped in overcoming hurdles by improving the ability to penetrate cells, effective and targeted binding to gene sequences, and downregulation of target gene function. Different delivery systems, including stable nucleic acid lipid particles, have shown potential in enhancing the delivery of cargo to the target site. In this review, we attempt to summarize the current progress in the development of antisense therapeutics and their potential in medical research. We partition this multicomponent review into introductory aspects about recent breakthroughs in antisense therapeutics. We also discuss how antisense therapeutics have shown potential in resensitizing resistant cancer cells to apoptosis by targeted inhibition of antiapoptotic proteins, oncogenic miRNAs, and BCR-ABL.
\end{abstract}

Keywords: antisense oligonucleotide, siRNA, miRNA, liposomes, DNAzymes, antisense therapy

\section{Introduction}

The application of RNAi therapy in the clinic requires safe and efficient carriers for the delivery of nucleic acids specifically to the target tissues and to the target cells. Currently, various physical and chemical methods are available for the transport of nucleic acids, which can be characterized into two subcategories, including viral vectors and nonviral vectors. Viral vector-based therapies typically exploit the viruses' intrinsic abilities to penetrate into the cells for the transport of nucleic acids. Nonviral strategies have different modes of action, either physical (electroporation, gene gun) or chemical (utilizing lipoplexes and polyplexes) for delivery. Viral vectors are advantageous because they have high transfection efficiencies. However their potential for mutagenesis, ability to induce host immune responses, and inability to carry large nucleic acid fragments are some of their frequently reported stumbling blocks. Lipoplexes (complexes of nucleic acids with cationic lipids) and polyplexes (complexes of nucleic acids with cationic polymers) have revolutionized nanotechnology because of their low toxicity and immunogenicity. 
However, a lower transfection rate is a challenge that must be addressed. Therefore, in recent years, advancements have been made in the field to increase transfer abilities and, hence, transfection efficiencies. The concept of gene therapy is older than the RNAi technology and, therefore, several methods have been optimized using nonviral vectors for the transfer of genes into cells. However, methods that are validated for gene delivery may not be equally efficient for RNAi technology. ${ }^{1}$ Therefore, separate nonviral strategies must be optimized for the transfer of small interfering RNA (siRNA) to inhibit or knockout expression of the gene of interest. There are exciting pieces of evidence substantiating efficient delivery of agents using liposomes and cationic polymers, including branched and linear polyethyleneimine. There are some characteristic features of each delivery system to ensure protection of the nucleic acid from degradation by endo- and exonucleases. However, the lack of endosomal escape and cargo release into the cytoplasm are some of the frequently noted challenges. "Naked" chemically modified siRNA has been successfully applied in treating certain diseases of the brain and lungs, as well as some others. ${ }^{2,3}$ Compared to systemic delivery, localized delivery of siRNA into target tissues and cells - for example, the eye, skin, lungs, and local tumors - offers several advantages, including high bioavailability and reduced side effects. For example, replication of respiratory syncytial virus in lungs was successfully inhibited using siRNA administered through the nasal cavity. ${ }^{4}$ Additionally, naked siRNA has also been used in subcutaneous cervical cancer mouse model treatments. ${ }^{5}$ However, the wide range of therapeutic applications of siRNA are limited by the potential for degradation by nucleases, reducing their half-life. Moreover, siRNA often activates an immunological response through the Toll-like receptor 7 (TLR7) pathway. ${ }^{6}$ Therefore, proper modifications of siRNA are required to increase their half-life and minimize their potential immunogenicity.

\section{Chemical modifications in siRNA}

Many chemical modifications have been made in siRNA, including modifications to the backbone of nucleobases, sugars, and termini of siRNA, to address the problems mentioned earlier. Common modifications to sugar moieties of siRNA are 2'-O-methyl, 2'-fluoro, and $2^{\prime}$-amine, and so on. ${ }^{7}$ For example, a $2^{\prime}$-O-methyl modification reduced the TLR7-induced immune response to siRNA, ${ }^{8,9}$ and it protected siRNA against endonuclease degradation. ${ }^{10}$ Similarly, nuclease resistance of siRNA can be increased by modifying siRNA with $2^{\prime}$-fluoro pyrimidines ${ }^{11}$ or by introducing phosphorothioate backbone linkages at the $3^{\prime}$-end of the
RNA strand. In addition, the therapeutic efficiency of siRNA can be enhanced with a corresponding decrease in off-target effects and nuclease sensitivity by modifying the backbone or terminal nucleotide of siRNA molecules. ${ }^{12}$ An example of the latter is cholesterol-modified siRNA that has an increased half-life and a corresponding biodistribution of siRNA to certain tissues. $^{13}$

However, apart from the tissues like the lungs, brain, and eye, there are several tissues that are not directly accessible to naked or modified siRNA alone and, therefore, siRNA requires different nanocarriers to deliver them to specific tissues in the body. Among nonviral nanocarriers, liposomes and lipid-derived vectors, as well as cationic polymers, have gained attention, although some other methods (such as gene gun, electroporation, and so on) are also used. ${ }^{14-17}$

The ability of liposomes to carry DNA fragments was demonstrated by Soriano et al in 1983. They reported that certain liposomes can transfer the preproinsulin gene into liver cells. Felgner et al showed for the first time that a cationic lipid DOTMA (N-[1-\{2,3-dioleyloxy $\}$ propyl]-N,N,Ntriethylammonium chloride) can potentially deliver nucleic acids, including DNA and RNA, into various cell lines. ${ }^{18,19}$ Subsequent studies enhanced gene delivery using various types of liposomes and lipid-derived vectors to improve delivery to specific tissues, minimize toxicity, side effects, and immunogenicity, and to protect nucleic acids from nuclease digestion. ${ }^{20,21}$ Recently, the discovery of the stable nucleic acid-lipid particles (SNALP) was applied in several in vivo models, including the inhibition of hepatitis $B$ virus (HBV) replication through siRNA-SNALP complexes targeted against HBV RNA and knockdown of alipoprotein B expression in cynomolgus monkeys' liver. ${ }^{22,23}$ Commercially available cationic liposomes, such as Lipofectamine ${ }^{\circledR} 2000$, Lipofectamine $^{\circledR}$, and Oligofectamine ${ }^{\mathrm{TM}}$ (Thermo Fisher Scientific, Waltham, MA, USA) have also been used to deliver siRNA in vitro and in vivo. For example, Huang ${ }^{24}$ efficiently suppressed the expression of keratoepithelin and myocilin using specific siRNA while using Lipofectamine as a delivery vehicle. Moreover, liposomes LipoTrust ${ }^{\mathrm{TM}}$ (Hokkaido System Science Co, Ltd, Hokkaido, Japan) and i-Fect ${ }^{\mathrm{TM}}$ (Neuromics, Edina, MN, USA) have been applied for the treatment of West Nile virus infection and liver cirrhosis in rats, respectively. ${ }^{25,26}$

Sequence-specific antisense oligonucleotides (AO) use different molecular mechanisms to exert their inhibitory effects, including messenger RNA (mRNA) translational arrest, alteration of mRNA splicing, and inducing degradation 
of targeted mRNA by RNase H. Like other RNA- and DNAderived therapeutics, there is progress in knowledge regarding the complexity of oligonucleotide pharmacology and modifications, including chemical modifications to the backbone, base, or sugar to enhance the properties of the drug, such as polysaccharide backbones, 2'-O-Me, 2'-F, 2'-MOE, and locked nucleic acid substitutions. Poly(N-(morpholino ethyl methacrylate)-based copolymers have emerged as versatile potential vehicles for the delivery of $\mathrm{AO}$ directed against the E6 gene of human papillomavirus. ${ }^{27}$ A combinatorial approach consisting of AO against laminin-411 and antibodycytokine fusion protein have shown encouraging results in immunocompetent mice bearing D2F2/E2 murine mammary tumors. ${ }^{28}$

DNAzymes are synthetic agents, which can bind and cleave mRNA of oncogenes, and they have proved to be effective. Notably, transfecting nasopharyngeal carcinoma cell line CNE2 with DNAzymes directed against the $E B V$ $L M P 1$ gene significantly repressed mRNA and protein expression of LMP1.29

\section{Stable nucleic acid lipid particles}

A new class of nucleic acid-lipid particles, designated SNALPs, may play a role in delivering molecules to a target site. F3-targeted SNALPs were internalized via nucleolin overexpressed on breast cancer cells. It was experimentally verified that delivery of anti-eGFP siRNA by F3-targeted liposomes considerably repressed mRNA and protein expression of eGFP in eGFP-overexpressing cancer cells. ${ }^{30}$ In vitro studies revealed wide ranging molecular mechanisms that underpin cancer progression, and it is now known that polo-like kinase 1 (PLK1) and kinesin spindle protein are overexpressed in various cancers. SNALP-formulated PLK1 siRNA induced the regression of tumor growth in mice inoculated with cancer cells. ${ }^{8,9}$ It has recently been convincingly revealed that targeted SNALPs with chlorotoxin as a ligand efficiently delivered anti-miR-21 oligonucleotides to glioblastoma multiforme/glioma cells. Xenografted mice injected with chlorotoxin-coupled SNALPs displayed considerably enhanced distribution in tissue homogenates. ${ }^{31}$

Next, we discuss recent advances in how AO have gained tremendous appreciation, as evidenced by the encouraging results obtained from in vitro and in vivo studies. We will provide an overview of recent updates on $\mathrm{AO}$ designed against oncogenes reported to trigger neoplastic processes such as cell migration and invasion, cell cycle and proliferation, and angiogenesis.

\section{Targeting of epidermal growth factor receptor}

Epidermal growth factor receptor (EGFR) is frequently overexpressed in different cancers and reported to be involved in processes ranging from cell proliferation to metastasis. Receptor-mediated intracellular signaling induced resistance against different therapeutic strategies.

\section{Cell proliferation}

EGF-induced signaling is involved in cellular proliferation. AO against EGFR encapsulated with poly(amidoamine) (PAMAM) nanoparticles have recently been shown to be effective in colon cancer cells. ${ }^{32}$ There was reduced expression of the EGFR-induced gene network in AO-treated cancer cells. Direct evidence suggests EGFR activation in the absence of growth factor, as evidenced by c-Src, a nonreceptor tyrosine kinase-mediated transactivation of EGFR. AO generated against c-Src complexed with PAMAM dendrimers has efficacy in inhibiting the transactivation of EGFR..$^{33}$ Dual targeting of EGFR and c-Src using PAMAM dendrimer-coated $\mathrm{AO}$ has also proved to be an effective approach, as evidenced by remarkably reduced expression of EGFR target genes in colon cancer cells. ${ }^{34}$ Gene silencing of different growth factors, including vascular endothelial growth factor (VEGF)-C, has also been tested in a lung carcinoma cell line. ${ }^{35} \mathrm{AO}$ directed against tumor necrosis factor (TNF)R1-associated death domain protein inhibited the proliferation potential of HepG2 cells in combination with proteasome inhibitors. ${ }^{36}$

It has been shown that LNCaP-derived MDV3100-resistant cell lines displayed a marked increase in clusterin expression. Detailed analysis revealed that androgen receptor (AR) pathway inhibition via MDV3100 resulted in an increase in the expression of clusterin. The underlying mechanism was also uncovered using different methodologies, and it was shown that MDV3100 induced activation of Akt, ERK, and p90RSK. p90RSK-mediated phosphoactivation of YB-1 stimulated the expression of clusterin. Notably, knockdown of clusterin using OGX-011 considerably enhanced MDV3100-induced LNCaP cell growth inhibition. ${ }^{37}$

\section{Metastasis and angiogenesis}

In gastric carcinoma SNU-5 and AGS cell lines, it has been shown that EGFR induced the activation of downstream proteins, including matrix metalloproteinase (MMP)7 and MMP13. Mechanistically, it was revealed that the PI3K/ ERK/MAPK signaling axis was involved in the activation of MMP7 and MMP13. ${ }^{38}$ EGFR-tyrosine kinase inhibitors 
in combination with an angiogenesis inhibitor worked with remarkable synergy by exerting inhibitory effects on angiogenesis in xenografted mice. ${ }^{39}$ BRCA2-targeting secondgeneration AOs significantly enhanced cisplatin-induced apoptosis in cancer cells. ${ }^{40} \mathrm{MGr} 1-\mathrm{Ag}$ is a precursor of the $37 \mathrm{kDa}$ laminin receptor and is frequently upregulated in a drug-resistant gastric carcinoma cell line. AO directed against MGr1-Ag/37LRP improved the efficacy of chemotherapeutic drugs in xenografted mice. ${ }^{41}$

\section{TRAIL-mediated signaling}

Data on TRAIL-mediated signaling has revolutionized the current understanding of different cancers. Detailed mechanistic insights have provided new dimensions in cancer, and there is a near complete resolution of positive and negative regulators of TRAIL-mediated signaling in cancer cells. Increasingly, it is being realized that stoichiometrically imbalanced ratios of proapoptotic and antiapoptotic proteins result in the impairment of TRAIL-induced apoptosis. Gene silencing strategies have shown potential in resensitizing resistant cancer cells to TRAIL-induced signals.

Several microRNAs (miRNAs) have been reported to regulate TRAIL-induced cell death, thus highlighting the fact that miRNAs may be a promising molecular target to reverse TRAIL resistance in cancer cells. Ovcharenko et $\mathrm{al}^{42}$ showed that miR-10a, miR-28, miR-196a, and miR-337 induced caspase- 3 activity, and that miR-96, miR-145, miR-150, miR-155, and miR-188 blocked TRAIL-induced caspase- 3 activation in MDA-MB-453 breast cancer cells. In particular, miR-96 and miR-145 have been predicted to interact with FAS-associated death domain and TRAIL-R1/2, respectively. ${ }^{42}$ The translational repression of oncogenic Six 1 transcriptional factor by miRNA-185 is associated with downregulation of c-Myc and cyclin A1, thus restoring apoptosis in TRAIL-resistant breast cancer cell lines. ${ }^{43}$ The reduction of SOC7 expression by mi-145 increases interferon (IFN) $\gamma$-dependent TRAIL expression and cell death in human bladder cancer cells. ${ }^{44}$ Several members of the miR-34 family are p53 targets, and their upregulation induces apoptosis and cell cycle arrest. ${ }^{45}$ Consequently, miR-34a and miR-34c increase TRAIL-induced apoptosis in lung cancer cells. ${ }^{46}$ Furthermore, miR-221 and miR-222 target PTEN and reduce TRAIL-induced apoptosis in lung and liver cancer. ${ }^{47,48}$ The miR-25 has been observed to target TRAIL-R1 expression, which induces resistance to cell death in cholangiocarcinoma. ${ }^{49} \mathrm{Mcl}-1$ is an antiapoptotic protein and it has been shown that overexpression of miR-29b reduced the level of Mcl-1 protein and sensitized cancer cells to TRAIL cytotoxicity in cholangiocarcinoma cell lines. ${ }^{50}$ Patron et $\mathrm{al}^{51}$ have demonstrated that miR-133b reduces the FAS apoptosis inhibitory molecule and promoted TRAILinduced cell death in vitro and in a xenograft-based prostate cancer cell model. Other studies demonstrated that miR-221 and miR-222 were markedly upregulated in TRAIL-resistant (Calu-1) and semiresistant (A459, A549) cells when compared to TRAIL-sensitive (H460) cells in nonsmall cell lung carcinoma (NSCLC) cells. ${ }^{47}$ This mechanism could be related to the observation that miR-221 silencing upregulated cyclin-dependent kinase inhibitor p27Kip1 and induced caspase- 3 in TRAIL-resistant bladder cancer T24 cells. ${ }^{52}$ miR-212 negatively regulates a death effector domain family member PED/PEA-15 and increased TRAIL-related sensitivity in NSCLC. ${ }^{53}$

For a targeted approach, researchers developed many strategies, including the development of vectors for the controlled expression of TRAIL. Recently, the inclusion of selected tumor downregulated miRNA response elements inserted immediately after the stop codon of the selected genes was noted to be effective in controlling the expression ofTRAIL in cancer cells when compared to the corresponding normal-counterpart cells. In this sense, the adenovirus-based gene therapy vector, including TRAIL and the combined miRNA response element sequence of tumor suppressors miRNA, miR-34a, miR-137, and miR-182, induced cell death in cultured uveal melanoma, but not in normal cell lines, as well as in a xenograft mouse model. ${ }^{54}$

\section{Anti-miRNA therapy}

It has been shown that miR-214 is upregulated in nasopharyngeal carcinoma. The results revealed that the subcutaneous injection of anti-miR-214-transfected CNE2 cells in nude mice inhibited tumorigenicity. ${ }^{55}$ Mounting evidence substantiates that silencing of oncogenic miRNAs in cancer cells restores sensitivity to chemotherapeutic drugs. In line with this approach, a recent experimental report indicated that miR-21 downregulation increased K562 and KYO-1 sensitivity to different chemotherapeutic drugs. miR-21 is involved in the negative regulation of autophagy-related proteins Vps34, beclin-1, and LC3-II. ${ }^{56}$ Systemically administered 2'-OMe$4^{\prime}$-thioribonucleoside-modified anti-miRNA directed against miR-122 was delivered efficiently to the liver and reduced the expression of miR-122. ${ }^{57}$ In line with this approach, another attempt was made using the systemic administration of an anti-miRNA oligonucleotide-encapsulated $\mathrm{pH}$-sensitive liposome, and the results revealed higher accumulation of anti-miRNA in the liver. ${ }^{58}$ 
Circumstantial evidence also highlighted considerably reduced xenograft growth of MCF-7 cells, as evidenced by subcutaneous injection of anti-miR-21 transfected MCF-7 cells in nude mice. ${ }^{59}$ Recently emerging technologies have shown potential in improving the delivery of cargo. Polyarginine-peptide nucleic acid (PNA) conjugates targeted against miR-221 have also been shown to significantly suppress miR-221 expression in MDA-MB-231 cells. ${ }^{60}$ Systemic delivery of anti-miR-9 in a xenograft model of Hodgkin lymphoma resulted in considerably reduced tumor outgrowth. ${ }^{61}$

\section{$B C R-A B L$ targeting using $A O$}

There is evidence from cutting-edge research indicating that there are approaches to inhibit BCR-ABL expression levels in leukemic cells. BCR-ABL modulates the transcriptional network of leukemic cells. It has been shown that VEGF expression is triggered by $\mathrm{BCR}-\mathrm{ABL}$, and that this results in the loss of sensitivity of leukemic cells to different drugs. Gene silencing of VEGF considerably improved the arsenic trioxide-mediated inhibitory effects on BCR-ABL in leukemia cells. ${ }^{62}$ Point mutations in BCR-ABL also add another layer of complication during the standardization of therapy, and attempts have been made to target mutant BCR-ABL using RNA-cleaving DNAzymes. PNAs (12mers) as facilitator oligonucleotides have shown remarkable potential in enhancing DNAzyme activity. Transfecting facilitator PNA and DNAzyme in drug-resistant leukemic cells dramatically improved the sensitivity of leukemic cells to imatinib. ${ }^{63}$ Antisense morpholino oligonucleotides have shown substantial potential in the translational inhibition of p210 (BCR$\mathrm{ABL}$ ) protein in K562 cells. ${ }^{64}$

\section{Antisense therapy in vivo}

Results have shown that the gene silencing of P55 protected mice against the toxicity of TNF/IFN $\gamma$ treatment in B16B16 and Lewis lung carcinoma cancer models. ${ }^{65}$ EZN-3920 is a locked nucleic acid-based HER3 AO that has been shown to effectively repress the HER3-modulated intracellular PI3K/ AKT signaling axis and expression of HER3. EZN-3920 in combination with either gefitinib or lapatinib remarkably reduced tumor growth in xenografted mice. ${ }^{66}$ Polyamidoamine dendrimer liposome-attached survivin AOs effectively reduced tumor growth in mice injected subcutaneously with SMMC7721 cells. $^{67}$ BRCA2-targeting second-generation AOs, in combination with cisplatin, reduced metastatic frequency in vivo by $77 \%{ }^{40}$ Mice xenografted with LNCaP cells displayed higher overall survival after treatment with MDV3100 and OGX-011. ${ }^{37}$ Chimeric CCL17 protein (TARC-arp) fused with the RNA-binding domain of HBV coupled with AO has recently been generated. TARC-arp has been shown to induce chemotaxis of cells expressing CCR4. siRNA-FoxP3 attached TARC-arp considerably inhibited FoxP3-expression in CCR4-expressing cells. BALB/c mice challenged with 4T1.2 breast cancer cells developed lung metastasis because of the involvement of $\mathrm{CCR} 4^{+} \mathrm{FoxP}^{+}$Tregs. Intravenous treatment of tumor-bearing mice seven times with $40 \mu \mathrm{g}$ of TARC-arp, coupled with $20 \mu \mathrm{g}$ of siRNA-FoxP3, dramatically suppressed the number of FoxP3 expressing T-cells and reduced metastatic foci in the lungs of mice. ${ }^{68}$

\section{Clinical trials}

EZN-4176 is a third-generation AO that binds to the hinge region (exon 4) of AR mRNA and degrades full-length AR mRNA. In a Phase I clinical trial, 22 patients were treated with EZN-4176 and the results revealed that the activity of EZN-4176 at the doses and schedules explored was minimal. The number of circulating tumor cells (CTC) in patients treated with EZN-4176 dropped from five or more CTC to less than five CTC in only three of eight patients. ${ }^{69}$

Different antiapoptotic proteins have also been considered, and gene silencing strategies have shown substantially enhanced response to therapeutics. AO sodium LY2181308 directed against survivin was tested in acute myeloid leukemia patients - first in a cohort with monotherapy - and it was shown that LY2181308 was well tolerated in patients with high survivin expression. A multipronged approach consisting of LY2181308, cytarabine, and idarubicin indicated that $4 / 16$ patients had complete responses. ${ }^{70}$

Custirsen, a second-generation AO, has recently been tested for efficacy in metastatic castration-resistant prostate cancer in combination with second-line chemotherapy in a Phase II trial. The findings of the study indicated a longer survival rate and serum clusterin was notably reduced in patients. ${ }^{71}$ Custirsen treatment was also found to be efficient in advanced NSCLC patients, as evidenced by decreased serum clusterin levels in $95 \%$ of patients evaluated during Phase I/II of the clinical trial. ${ }^{72}$

LY2181308 is a survivin AO that failed to provide encouraging results in combination with first-line docetaxel/ prednisone in castration-resistant prostate cancer patients. Patients had a higher incidence of anemia, thrombocytopenia, and grades 3-4 neutropenia. ${ }^{73}$ EZN-2968 is an AO designed to specifically target HIF- $1 \alpha$ and was given as a 2 -hour intravenous infusion at a dose of $18 \mathrm{mg} / \mathrm{kg}$ once a week to patients with refractory advanced solid tumors. Four of six patients displayed a decrease in HIF- $1 \alpha$ mRNA levels. ${ }^{74}$ 
Oblimersen is a Bcl-2 AO that has been noted to be effective in patients with advanced melanoma when used in combination with albumin-bound paclitaxel and temozolomide. The response rate was $40.6 \%$ : eleven patients had a partial response; two patients had a complete response; and eleven patients had stable disease. ${ }^{75}$ The STAT3 decoy displayed significant pharmacodynamic activity in head and neck squamous cell carcinoma patients who received a single intratumoral injection of STAT3 decoy. ${ }^{76}$

\section{Conclusion}

RNAi therapeutics offers applications in many cancers and several diseases. However, the lack of ideal delivery methods hampers therapeutic applications. Nonviral vectors offer an alternative for the delivery of siRNA; however, they lag behind their viral counterparts in transfection efficiency. Finding efficient ways to design nanocarriers with the abilities to cross cellular barriers in an efficient manner with corresponding lower toxicity and fewer off-target effects will make RNAi therapeutics possible.

\section{Disclosure}

The authors report no conflicts of interest in this work.

\section{References}

1. Scholz C, Wagner E. Therapeutic plasmid DNA versus siRNA delivery: common and different tasks for synthetic carriers. J Control Release. 2012;161(2):554-565.

2. DiFiglia M, Sena-Esteves M, Chase K, et al. Therapeutic silencing of mutant huntingtin with siRNA attenuates striatal and cortical neuropathology and behavioral deficits. Proc Natl Acad Sci U SA. 2007;104(43): 17204-17209.

3. de Fougerolles A, Novobrantseva T. siRNA and the lung: research tool or therapeutic drug? Curr Opin Pharmacol. 2008;8(3):280-285.

4. Bitko V, Musiyenko A, Shulyayeva O, Barik S. Inhibition of respiratory viruses by nasally administered siRNA. Nat Med. 2005;11(1):50-55.

5. Niu XY, Peng ZL, Duan WQ, Wang H, Wang P. Inhibition of HPV $16 \mathrm{E} 6$ oncogene expression by RNA interference in vitro and in vivo. Int J Gynecol Cancer. 2006;16(2):743-751.

6. Hornung V, Guenthner-Biller M, Bourquin C, et al. Sequence-specific potent induction of IFN-alpha by short interfering RNA in plasmacytoid dendritic cells through TLR7. Nat Med. 2005;11(3):263-270.

7. Chiu YL, Rana TM. siRNA function in RNAi: a chemical modification analysis. RNA. 2003;9(9):1034-1048.

8. Judge AD, Robbins M, Tavakoli I, et al. Confirming the RNAi-mediated mechanism of action of siRNA-based cancer therapeutics in mice. J Clin Invest. 2009;119(3):661-673.

9. Judge AD, Bola G, Lee AC, MacLachlan I. Design of noninflammatory synthetic siRNA mediating potent gene silencing in vivo. Mol Ther. 2006;13(3):494-505.

10. Vornlocher HP, Hadwiger P, Zimmerman T, et al, inventor; Alnylam Pharmaceuticals, Inc., assignee. Nuclease-resistant double-stranded RNA for RNA interference. United States patent WO 2005115481. August 12, 2005.

11. Layzer JM, McCaffrey AP, Tanner AK, Huang Z, Kay MA, Sullenger BA. In vivo activity of nuclease-resistant siRNAs. RNA. 2004;10(5): 766-771.
12. Terrazas M, Kool ET. RNA major groove modifications improve siRNA stability and biological activity. Nucleic Acids Res. 2009;37(2): 346-353.

13. Soutschek J, Akinc A, Bramlage B, et al. Therapeutic silencing of an endogenous gene by systemic administration of modified siRNAs. Nature. 2004;432(7014):173-178.

14. Xu Y, Szoka FC Jr. Mechanism of DNA release from cationic liposome/DNA complexes used in cell transfection. Biochemistry. 1996;35(18):5616-5623.

15. Torchilin VP. Recent advances with liposomes as pharmaceutical carriers. Nat Rev Drug Discov. 2005;4(2):145-160.

16. Boussif O, Lezoualc' $h \mathrm{~F}$, Zanta MA, et al. A versatile vector for gene and oligonucleotide transfer into cells in culture and in vivo: polyethylenimine. Proc Natl Acad Sci U SA. 1995;92(16):7297-7301.

17. Putnam D. Polymers for gene delivery across length scales. Nat Mater. 2006;5(6):439-451.

18. Soriano P, Dijkstra J, Legrand A, et al. Targeted and nontargeted liposomes for in vivo transfer to rat liver cells of a plasmid containing the preproinsulin I gene. Proc Natl Acad Sci U S A. 1983;80(23):7128-7131.

19. Felgner PL, Gadek TR, Holm M, et al. Lipofection: a highly efficient, lipid-mediated DNA-transfection procedure. Proc Natl Acad Sci U SA. 1987;84(21):7413-7417.

20. Schroeder A, Levins CG, Cortez C, Langer R, Anderson DG. Lipid-based nanotherapeutics for siRNA delivery. J Intern Med. 2010;267(1):9-21.

21. Rehman Zu, Zuhorn IS, Hoekstra D. How cationic lipids transfer nucleic acids into cells and across cellular membranes: recent advances. J Control Release. 2013;166(1):46-56.

22. Morrissey DV, Lockridge JA, Shaw L, et al. Potent and persistent in vivo anti-HBV activity of chemically modified siRNAs. Nat Biotechnol. 2005;23(8):1002-1007.

23. Zimmermann TS, Lee AC, Akinc A, et al. RNAi-mediated gene silencing in non-human primates. Nature. 2006;441(7089):111-114.

24. Huang AJ. Suppression of keratoepithelin and myocilin by small interfering RNA (an American Ophthalmological Society thesis). Trans Am Ophthalmol Soc. 2007;105:365-378.

25. Sato Y, Murase K, Kato J, et al. Resolution of liver cirrhosis using vitamin A-coupled liposomes to deliver siRNA against a collagenspecific chaperone. Nat Biotechnol. 2008;26(4):431-442.

26. Kumar P, Lee Sk, Shankar P, Manjunath N. A single siRNA suppresses fatal encephalitis induced by two different flaviviruses. PLoS Med. 2006;3(4):e96.

27. Reschner A, Shim YH, Dubois P, et al. Evaluation of a new biocompatible poly(N-(morpholino ethyl methacrylate)-based copolymer for the delivery of ruthenium oligonucleotides, targeting HPV16 E6 oncogene. J Biomed Nanotechnol. 2013;9(8):1432-1440.

28. Ding H, Helguera G, Rodríguez JA, et al. Polymalic acid nanobioconjugate for simultaneous immunostimulation and inhibition of tumor growth in HER2/neu-positive breast cancer. $J$ Control Release. 2013;171(3):322-329.

29. You X, Yang YC, Ke X, Hong SL, Hu GH. Fluorescence visualization screening for EBV-LMP1-targeted DNAzymes. Otolaryngol Head Neck Surg. 2014;150(2):251-258.

30. Gomes-da-Silva LC, Santos AO, Bimbo LM, et al. Toward a siRNAcontaining nanoparticle targeted to breast cancer cells and the tumor microenvironment. Int J Pharm. 2012;434(1-2):9-19.

31. Costa PM, Cardoso AL, Mendonça LS, et al. Tumor-targeted chlorotoxin-coupled nanoparticles for nucleic acid delivery to glioblastoma cells: a promising system for glioblastoma treatment. Mol Ther Nucleic Acids. 2013;2:e100.

32. Najar AG, Pashaei-Asl R, Omidi Y, Farajnia S, Nourazarian AR. EGFR antisense oligonucleotides encapsulated with nanoparticles decrease EGFR, MAPK1 and STAT5 expression in a human colon cancer cell line. Asian Pac J Cancer Prev. 2013;14(1):495-498.

33. Nourazarian AR, Najar AG, Farajnia S, Khosroushahi AY, Pashaei-Asl R, Omidi Y. Combined EGFR and c-Src antisense oligodeoxynucleotides encapsulated with PAMAM denderimers inhibit HT-29 colon cancer cell proliferation. Asian Pac J Cancer Prev. 2012;13(9):4751-4756. 
34. Nourazarian AR, Pashaei-Asl R, Omidi Y, Najar AG. c-Src antisense complexed with PAMAM denderimes decreases of c-Src expression and EGFR-dependent downstream genes in the human HT-29 colon cancer cell line. Asian Pac J Cancer Prev. 2012;13(5): 2235-2240.

35. Peng C, Sun Q, Fang Y, Cong B, Zhao X. VEGF-C antisense oligoxydenucleotide suppression of invasive ability of the A-549 lung carcinoma cell line. Asian Pac J Cancer Prev. 2011;12(8):2097-2099.

36. Witort E, Lulli M, Carloni V, Capaccioli S. Anticancer activity of an antisense oligonucleotide targeting TRADD combined with proteasome inhibitors in chemoresistant hepatocellular carcinoma cells. J Chemother. 2013;25(5):292-297.

37. Matsumoto H, Yamamoto Y, Shiota M, et al. Cotargeting androgen receptor and clusterin delays castrate-resistant prostate cancer progression by inhibiting adaptive stress response and AR stability. Cancer Res. 2013;73(16):5206-5217.

38. Ye Y, Zhou X, Li X, Tang Y, Sun Y, Fang J. Inhibition of epidermal growth factor receptor signaling prohibits metastasis of gastric cancer via downregulation of MMP7 and MMP13. Tumour Biol. Epub August 2, 2014.

39. Ito K, Semba T, Uenaka T, Wakabayashi T, Asada M, Funahashi Y. Enhanced anti-angiogenic effect of E7820 in combination with erlotinib in epidermal growth factor receptor-tyrosine kinase inhibitorresistant non-small-cell lung cancer xenograft models. Cancer Sci. 2014;105(8):1023-1031.

40. Rytelewski M, Tong JG, Buensuceso A, et al. BRCA2 inhibition enhances cisplatin-mediated alterations in tumor cell proliferation, metabolism, and metastasis. Mol Oncol. Epub June 6, 2014. pii: S15747891(14)00124-00120.

41. Sun L, Liu L, Liu X, et al. MGr1-Ag/37LRP induces cell adhesionmediated drug resistance through FAK/PI3K and MAPK pathway in gastric cancer. Cancer Sci. 2014;105(6):651-659.

42. Ovcharenko D, Kelnar K, Johnson C, Leng N, Brown D. Genomescale microRNA and small interfering RNA screens identify small RNA modulators of TRAIL-induced apoptosis pathway. Cancer Res. 2007;67(22):10782-10788.

43. Imam JS, Buddavarapu K, Lee-Chang JS, et al. MicroRNA-185 suppresses tumor growth and progression by targeting the Six 1 oncogene in human cancers. Oncogene. 2010;29(35):4971-4979.

44. Noguchi S, Yamada N, Kumazaki M, et al. socs7, a target gene of microRNA-145, regulates interferon- $\beta$ induction through STAT3 nuclear translocation in bladder cancer cells. Cell Death Dis. 2013;4:e482.

45. He L, He X, Lim LP, et al. A microRNA component of the p53 tumour suppressor network. Nature. 2007;447(7148):1130-1134.

46. Garofalo M, Jeaon YJ, Nuovo GJ, et al. MiR-34a/c-dependent PDGFR$\alpha / \beta$ downregulation inhibits tumorigenesis and enhances TRAILinduced apoptosis in lung cancer. PLoS One. 2013;8(6):e67581.

47. Garofalo M, Quintavalle C, Di Leva G, et al. MicroRNA signatures of TRAIL resistance in human non-small cell lung cancer. Oncogene. 2008;27(27):3845-3855.

48. Garofalo M, Di Leva G, Romano G, et al. mir-221 and 222 regulate TRAIL resistance and enhance tumorigenicity through PTEN and TIMP3 downregulation. Cancer Cell. 2009;16(6):498-509.

49. Razumilava N, Bronk SF, Smoot RL, et al. miR-25 targets TNF-related apoptosis inducing ligand (TRAIL) death receptor-4 and promotes apoptosis resistance in cholangiocarcinoma. Hepatology. 2012;55(2): 465-475.

50. Mott JL, Kobayashi S, Bronk SF, Gores GJ. mir-29 regulates Mcl-1 protein expression and apoptosis. Oncogene. 2007;26(42): 6133-6140.

51. Patron JP, Fendler A, Bild M, et al. MiR-133b targets antiapoptotic genes and enhances death receptor-induced apoptosis. PLoS One. 2012;7(4):e35345.

52. Lu Q, Lu C, Zhou GP, Zhang W, Xiao H, Wang XR. MicroRNA-221 silencing predisposed human bladder cancer cells to undergo apoptosis induced by TRAIL. Urol Oncol. 2010;28(6):635-641.
53. Incoronato $\mathrm{M}$, Garofalo $\mathrm{M}$, Urso $\mathrm{L}$, et al. miR-212 increases tumor necrosis factor-related apoptosis-inducing ligand sensitivity in nonsmall cell lung cancer by targeting the antiapoptotic protein PED. Cancer Res. 2010;70(9):3638-3646.

54. Liu J, Ma L, Li C, Zhang Z, Yang G, Zhang W. Tumor-targeting TRAIL expression mediated by miRNA response elements suppressed growth of uveal melanoma cells. Mol Oncol. 2013;7(6):1043-1055.

55. Zhang ZC, Li YY, Wang HY, et al. Knockdown of miR-214 promotes apoptosis and inhibits cell proliferation in nasopharyngeal carcinoma. PLoS One. 2014;9(1):e86149.

56. Seca H, Lima RT, Lopes-Rodrigues V, Guimaraes JE, Almeida GM, Vasconcelos MH. Targeting miR-21 induces autophagy and chemosensitivity of leukemia cells. Curr Drug Targets. 2013;14(10): 1135-1143.

57. Takahashi M, Yamada N, Hatakeyama $\mathrm{H}$, et al. In vitro optimization of 2'-OMe-4'-thioribonucleoside-modified anti-microRNA oligonucleotides and its targeting delivery to mouse liver using a liposomal nanoparticle. Nucleic Acids Res. 2013;41(22):10659-10667.

58. Hatakeyama H, Murata M, Sato Y, et al. The systemic administration of an anti-miRNA oligonucleotide encapsulated $\mathrm{pH}$-sensitive liposome results in reduced level of hepatic microRNA-122 in mice. J Control Release. 2014;173:43-50.

59. Yan LX, Wu QN, Zhang Y, et al. Knockdown of miR-21 in human breast cancer cell lines inhibits proliferation, in vitro migration and in vivo tumor growth. Breast Cancer Res. 2011;13(1):R2.

60. Brognara E, Fabbri E, Aimi F, et al. Peptide nucleic acids targeting miR-221 modulate p27Kip1 expression in breast cancer MDA-MB-231 cells. Int J Oncol. 2012;41(6):2119-2127.

61. Leucci E, Zriwil A, Gregersen LH, et al. Inhibition of miR-9 derepresses HuR and DICER1 and impairs Hodgkin lymphoma tumour outgrowth in vivo. Oncogene. 2012;31(49):5081-5089.

62. Luo X, Feng M, Zhu X, Li Y, Fei J, Zhang Y. VEGF depletion enhances bcr-abl-specific sensitivity of arsenic trioxide in chronic myelogenous leukemia. Hematology. 2013;18(6):334-340.

63. Kim JE, Yoon S, Mok H, Jung W, Kim DE. Site-specific cleavage of mutant ABL mRNA by DNAzyme is facilitated by peptide nucleic acid binding to RNA substrate. FEBS Lett. 2012;586(21):3865-3869.

64. Haririan M, Kaviani S, Soleimani M, Ghaemi SR, Delalat B, Atashi A. Morpholino oligo antisense efficiently suppresses BCR/ABL and cell proliferation in CML: specific inhibition of BCR-ABL gene expression by morpholino oligo antisense in BCR-ABL(+) cells. Hematology. 2012;17(1):28-34

65. Van Hauwermeiren F, Vandenbroucke RE, Grine L, et al. Antisense oligonucleotides against TNFR1 prevent toxicity of TNF/IFN $\gamma$ treatment in mouse tumor models. Int J Cancer. 2014;135(3):742-750.

66. Wu Y, Zhang Y, Wang M, et al. Downregulation of HER3 by a novel antisense oligonucleotide, EZN-3920, improves the antitumor activity of EGFR and HER2 tyrosine kinase inhibitors in animal models. Mol Cancer Ther. 2013;12(4):427-437.

67. Han S, Cai Z, Peng L, et al. Polyamidoamine dendrimer liposome-mediated survivin antisense oligonucleotide inhibits hepatic cancer cell proliferation by inducing apoptosis. Tumour Biol. 2014;35(5):5013-5019.

68. Biragyn A, Bodogai M, Olkhanud PB, et al. Inhibition of lung metastasis by chemokine CCL17-mediated in vivo silencing of genes in CCR4+ Tregs. J Immunother. 2013;36(4):258-267.

69. Bianchini D, Omlin A, Pezaro C, et al. First-in-human Phase I study of EZN-4176, a locked nucleic acid antisense oligonucleotide to exon 4 of the androgen receptor mRNA in patients with castration-resistant prostate cancer. Br J Cancer. 2013;109(10):2579-2586.

70. Erba HP, Sayar H, Juckett M, et al. Safety and pharmacokinetics of the antisense oligonucleotide (ASO) LY2181308 as a single-agent or in combination with idarubicin and cytarabine in patients with refractory or relapsed acute myeloid leukemia (AML). Invest New Drugs. 2013;31(4):1023-1034.

71. Blumenstein B, Saad F, Hotte S, et al. Reduction in serum clusterin is a potential therapeutic biomarker in patients with castration-resistant prostate cancer treated with custirsen. Cancer Med. 2013;2(4):468-477. 
72. Laskin JJ, Nicholas G, Lee C, et al. Phase I/II trial of custirsen (OGX-011), an inhibitor of clusterin, in combination with a gemcitabine and platinum regimen in patients with previously untreated advanced non-small cell lung cancer. J Thorac Oncol. 2012;7(3):579-586.

73. Wiechno P, Somer BG, Mellado B, et al. A randomised phase 2 study combining LY2181308 sodium (survivin antisense oligonucleotide) with first-line docetaxel/prednisone in patients with castration-resistant prostate cancer. Eur Urol. 2014;65(3):516-520.

74. Jeong W, Rapisarda A, Park SR, et al. Pilot trial of EZN-2968, an antisense oligonucleotide inhibitor of hypoxia-inducible factor-1 alpha (HIF-1 $\alpha$ ), in patients with refractory solid tumors. Cancer Chemother Pharmacol. 2014;73(2):343-348.
75. Ott PA, Chang J, Madden K, et al. Oblimersen in combination with temozolomide and albumin-bound paclitaxel in patients with advanced melanoma: a phase I trial. Cancer Chemother Pharmacol. 2013;71(1): 183-191.

76. Sen M, Thomas SM, Kim S, et al. First-in-human trial of a STAT3 decoy oligonucleotide in head and neck tumors: implications for cancer therapy. Cancer Discov. 2012;2(8):694-705.

\section{Publish your work in this journal}

OncoTargets and Therapy is an international, peer-reviewed, open access journal focusing on the pathological basis of all cancers, potential targets for therapy and treatment protocols employed to improve the management of cancer patients. The journal also focuses on the impact of management programs and new therapeutic agents and protocols on

\section{Dovepress}

patient perspectives such as quality of life, adherence and satisfaction The manuscript management system is completely online and includes a very quick and fair peer-review system, which is all easy to use. Visit http://www.dovepress.com/testimonials.php to read real quotes from published authors.

\footnotetext{
Submit your manuscript here: http://www.dovepress.com/oncotargets-and-therapy-journal
} 